\title{
The least squares reverse time migration in frequency domain
}

\author{
Lv Yin-yin \\ College of GeoEexploration Science and Technology \\ Jilin University \\ Changchun, China \\ e-mail: 112003303@qq.com
}

Xu Shi

College of GeoEexploration Science and Technology Jilin University

Changchun, China

e-mail: xushiyx@163.com

\author{
Chen Xiong \\ College of GeoEexploration Science and Technology \\ Jilin University \\ Changchun, China \\ e-mail: 412680936@qq.com
}

\author{
$\mathrm{Hu}$ Ying-cai \\ College of GeoEexploration Science and Technology \\ Jilin University \\ Changchun, China \\ e-mail:406252759@qq.com
}

\begin{abstract}
Firstly, from the point of view of the frequency domain Helmholtz equation, a detailed description of the frequency domain modeling process and frequency domain blended seismic acquisition forward process were given. By solving the Helmholtz equation, reverse-time migration method in frequency domain is derived. Reverse time migration method based on frequency domain, the frequency domain least-squares reverse-time migration technology. Based on the Marmousi model, the typical representative, through the method of mixing the offset frequency domain and achieved good results. Comparative analysis of the factors of the frequency domain least-squares reverse-time migration effect and advantages and disadvantages.
\end{abstract}

Keywords- multi-source simultaneous acquisition; multisource blended acquisition; reverse-time migration; leastsquares reverse-time migration; frequency domain Helmholtz equation;

\section{INTRODUCTION}

Seismic acquisition is a trade-off between economic and quality. Conventional acquisition surveys are designed such that the time intervals between successive shots are sufficiently large to avoid the interference between different source responses. However in this situation both the image quality and measure parameters, such as azimuths and sampling density may not be achieve the best. Unfortunately the improvement of seismic acquisition

\author{
Liu Zong-li \\ College of GeoEexploration Science and Technology \\ Jilin University \\ Changchun, China \\ e-mail: 953978142@qq.com
}

\author{
Mou Dan \\ College of GeoEexploration Science and Technology \\ Jilin University \\ Changchun, China \\ e-mail:mudan-main@163.com
}

\author{
Zhang Xue-ang \\ College of GeoEexploration Science and Technology \\ Jilin University \\ Changchun, China \\ e-mail: 625014321@qq.com
}

\author{
Li Jing \\ College of GeoEexploration Science and Technology \\ Jilin University \\ Changchun, China \\ e-mail: 496443960@qq.com
}

method is fall behind other technologies in seismic processing. Recently a great change of mindset in seismic acquisition has occurred which is known as blended acquisition or simultaneous acquisition, where different sources at different locations are shot in an overlapping fashion. Both which significantly improved the acquisition efficiency and potentially image quality can be significantly improved.

Multi-source simultaneous acquisition has brought a revolution of seismic data acquisition methods, from which many seismic data processing methods will benefit. This acquisition method is applied to the virtual source method. Compare the virtual source gathers constructed by the conventional acquisition method and the simultaneous method we can find that: the virtual source records builded from the separation results of blended data are much more closed to the synthetic ones. Based on the simulated data are tested and compared with other noise suppression methods, show that this method can effectively.

\section{SEISMIC DATA FORWARD IN FREQUENCY DOMAIN}

Fourier transform can be scalar wave equation in frequency domain to the time domain of two order scalar wave equation (Helmholtz equation).

$$
A u=-\nabla^{2} u-k^{2} u=g
$$

$\nabla^{2}$ is Laplace operator, $A=-\nabla^{2}-k^{2}$ is Helmholtz operator, $u$ is displacement, $k=\omega / c$ is the wave number 
( $\omega$ is the angular frequency, $c$ is medium speed), $g$ is the source terms.

With an attenuation factor, (1) can be written as:

$$
A u=-\nabla^{2} u-(1-\beta j) k^{2} u=g .
$$

$0 \leq \beta \leq 1$ is an attenuation factor of the actual needs. When $\beta=0$, equation (2) is equal to equation (1), and equation (2) used on the boundary, can be see a wave field attenuation method.

\section{A. Frequency Domain Modelingt}

1 to $\mathrm{n}$ is the source sequence number of single source $S$, the size of source terms matrices is $N \times n$. As shown in figure 1 , the conventional single source simulation can be rewritten as follows:

$$
\underset{N \times n}{P}=B_{N \times N}^{-1} \cdot \underset{N \times n}{S}
$$

Fig.1b shows the source coding method. In the diagram, source were divided into three groups, such as the code matrix $\Gamma$, the size of the code matrices is $n \times l$. After coding, the dimension of source matrix reduced greatly, as shown in $S \Gamma^{T}$. This process can be expressed by the formula:

$$
\underset{N \times l}{S_{b}}=\underset{N \times n}{S} \cdot \Gamma_{n \times l}^{T}
$$

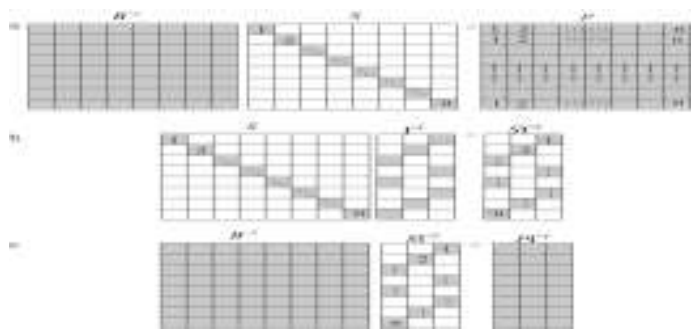

Figure 1. conventional single shot and multi shots forward in frequency domain

Fig.1c shows the forward modeling after encoded. We think the source code is a super source vector, then the multi shots forward wave field also can be see a vector. So Fig.1c using the following formula:

$$
\underset{N \times l}{P_{b}}=\underset{N \times n}{P} \cdot \Gamma_{n \times l}^{T}=\underset{N \times N}{B^{-1}} \cdot \underset{N \times l}{S_{b}}=\underset{N \times N}{B^{-1}} \cdot \underset{N \times n}{S} \cdot \Gamma_{n \times l}^{T}
$$

From equation (5), produce multi shots forward record has two forms: direct to code the single shot forward record, such as $\underset{N \times n}{P \cdot \Gamma_{n \times l}^{T}}$; and encode the source, such as $\underset{N \times n}{S} \cdot \Gamma_{n \times l}^{T}$. Here, we used the second encod method.

According the different of the encoding function $\Gamma$, the forward result is different. These can be divided into: the simultaneous acquisition; the mixed delay acquisition technology. Firstly,the encoding function description as follows:

$$
\begin{aligned}
& \Gamma=\left[a_{1} \gamma_{1}, a_{2} \gamma_{2}, \ldots, a_{n} \gamma_{n}\right]^{T} \\
& \gamma_{i}=e^{-j \omega \Delta t_{i}}, i=1,2, \ldots, n
\end{aligned}
$$

the $\gamma_{i}$ is delay function, the $a_{i}$ is random amplitude, the $\omega$ is the angular frequency, the $\Delta t_{i}$ is delay time.

When $\gamma_{i}$ is a constant, the $\Delta t_{i}$ is constant, then all the source will be excited at the same time, the encoded source for simultaneous excitation source. If the random amplitude ${ }^{a_{i}}$ is a constant, so the excitation source is also one by one single shot repeatedly by the replication. This kind of situation is not only inconsistent with the actual and the cross term of energy is very big. In this paper, the amplitude is random, that is the so-called random amplitude coding and excitation source.

When $\gamma_{i}$ is a random number, the $\Delta t_{i}$ is random delay blasting time. Thus the source is the random delay of excitation, seismic data acquisition is also a mixing source. The random coding mode is multi shots at the same time collection technology is compared with the field excited at the same time acquisition. Compared with the simultaneous excitation of acquisition technology, random delay excitation delay data acquisition time, avoid and strong amplitude blasting, improve the proportion of effective information

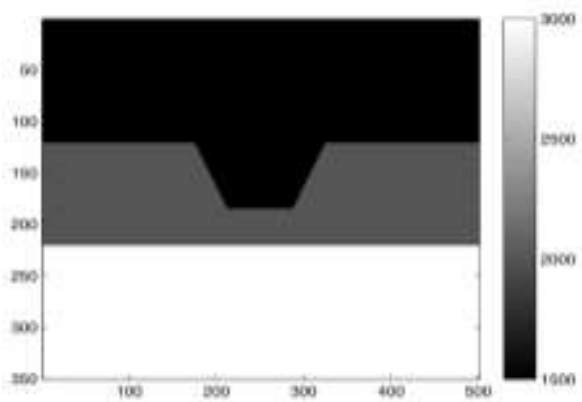

Figure 2. velocity mode

The model 1 as shown in Fig .2, the three layers of medium speed is $v 1=1500 \mathrm{~m} / \mathrm{s}, \quad v 2=2000 \mathrm{~m} / \mathrm{s}, \quad v 3=3000 \mathrm{~m} / \mathrm{s}$ respectively, and the source in the deep $50 \mathrm{~m}$, laterally $1250 \mathrm{~m}$. Frequency sampling interval is $0.42 \mathrm{~Hz}, 101$ frequency sampling points, the maximum frequency is $42 \mathrm{~Hz}$

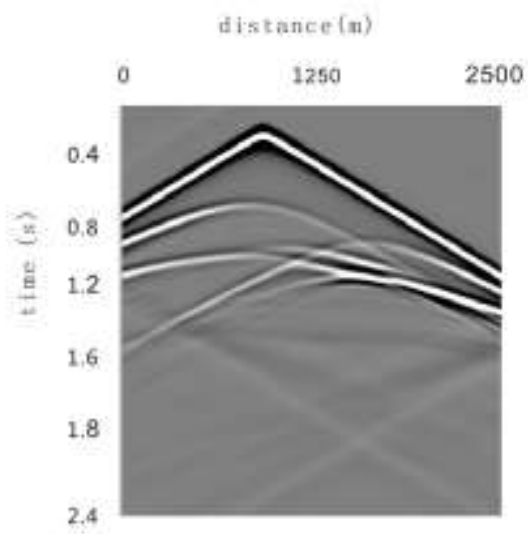

Figure 3. frequency domain forward record

Igneous rock mineral composition and Compared with the conventional migration method,the least squares migration can improve the imaging quality, but in the practical application, the calculation of the least squares is huge [2]. In this paper, in order to improve the computational efficiency of the least square, we use the reverse time migration algorithm and hybrid acquisition technology into the least squares migration. Through the iterative migration algorithm[3], we can improve the image quality,and the crosstalk noise produced by the 
source mixing have been effectively suppressed too. In the model test, we found that the least-squares migration algorithm can suppress the migration noise, improves the image resolution, and reduces crosstalk noise produced by mixed sources introduced. Compared with the reverse time migration,the multi sourses least-squares reverse time migration not only improves the computing speed,the imaging quality is also effectively promote [4].

\section{LEAST SQUARES MIGRATION IN FREQUENCY DOMAIN}

The operator of Helmholtz equations, we can use the Born approximate,then the forward modeling is a linear inversion, when we do not use the Born approximation, the seismic inversion is a quasi linear inversion [5].

For the background model ${ }^{s_{0}}$, the Green function of the Helmholtz equation can be obtained by solution the following equation:

$$
\left[\nabla^{2}+\omega^{2} s_{0}(x)^{2}\right] G_{0}\left(x \mid x_{s}\right)=-\delta\left(x-x_{s}\right) .
$$

the $x_{s}$ is a point source position, the $x$ is detector, the $\omega$ is angular frequency, the ${ }^{G_{0}\left(x x_{s}\right)}$ is Green function. For a point source ${ }^{X_{s}}$, the $W(\omega)$ is corresponding source $\left.F=(x, \omega)=-\delta\left(x-x_{s}\right) W(\omega) \quad\right)$, the solution is $P_{0}\left(x \mid X_{s}\right)=W(\omega) G_{0}\left(x \mid X_{s}\right)$

If the background disturbance is $\delta s(x)$, the actual background model is $S(x)=s_{0}+\delta S(x)$, solution the Helmholtz equations,

$$
\left[\nabla^{2}+\omega^{2} s(x)^{2}\right] P=F .
$$

take $S(x)=s_{0}+\delta S(x)$ into equation 8 , can be obtained:

$$
\left[\nabla^{2}+\omega^{2} s_{0}(x)^{2}+2 \omega^{2} s(x)_{0} \delta s(x)\right] P=F
$$

The higher order terms $O\left(\delta s^{2}\right)$ can be neglected. According to Green's ,we move the third of the left side in equation 9 to the right, and the two sides multiplied by the Green function at the same time, and the integral equation:

$$
\begin{aligned}
P(x) & =\int G_{0}\left(x \mid x^{\prime}\right) F\left(x^{\prime}\right) d x^{\prime}-2 \omega^{2} \int s\left(x^{\prime}\right)_{0} \delta s\left(x^{\prime}\right) P\left(x^{\prime} \mid x_{s}\right) G_{0}\left(x \mid x^{\prime}\right) d x^{\prime} \\
& =P_{0}(x)+\omega^{2} \int m\left(x^{\prime}\right) P\left(x^{\prime} \mid x_{s}\right) G_{0}\left(x \mid x^{\prime}\right) d x^{\prime}
\end{aligned}
$$

The $P_{0}(x)$ is the pressure field of background velocity model, both sides of the equation containing the $P(x)$, the $m\left(x^{\prime}\right)$ is reflection model. At the same time, the Born approximation $G\left(x^{\prime} \mid x_{s}\right) \approx G_{0}\left(x^{\prime} \mid x_{s}\right)$, assuming that the $\delta s(x)$ perturbation is small, then the Born approximation for scattering field:

$$
\begin{aligned}
P_{1}= & P(x)-P_{0}(x) \\
& \approx \omega^{2} \int W(\omega) m\left(x^{\prime}\right) G_{0}\left(x^{\prime} \mid x_{s}\right) G_{0}\left(x \mid x^{\prime}\right) d x^{\prime},
\end{aligned}
$$

Equation (10) is a nonlinear equation to calculate the scattering field, equation (11) is a linear equation, when $P_{0}\left(x^{\prime}\right)=W(\omega) G_{0}\left(x^{\prime} \mid x_{s}\right)$, the linear simulation needs to solve the following equation:

$$
\begin{aligned}
& {\left[\nabla^{2}+\omega^{2} s_{0}(x)^{2}\right] P_{0}=F} \\
& {\left[\nabla^{2}+\omega^{2} s_{0}(x)^{2}\right] P_{1}=\omega^{2} m(x) P_{0}\left(x^{\prime}\right) .}
\end{aligned}
$$

All of these can use 2 finite difference simulation calculation: first, using point source $\mathrm{F}$ and velocity model to obtain $P_{0}$; the second finite difference background model also is $S_{0}$, but the source function is $\omega^{2} m\left(x^{\prime}\right) P_{0}\left(x^{\prime}\right)$ ( $\omega^{2}$ is 2 order time domain differential). Linear operators with matrix is reverse time migration operator, so reverse time migration equation:

$$
m_{\text {mig }}(x)=\sum_{X_{s}} \int \omega^{2} W^{*}(\omega) P_{1}\left(x^{\prime}\right) G_{0}^{*}\left(x \mid x_{s}\right) G_{0}^{*}\left(x \mid x^{\prime}\right) d x^{\prime} .
$$

Use the matrix to define the operator, we can get the nonlinear operator $\mathrm{A}: \mathrm{d}=\mathrm{A}(m) P-P_{0}$, the linear operator $\mathrm{L}: \mathrm{d}=\mathrm{Lm}$, the reverse time migration operator is $L^{T}: \mathrm{m}=L^{T} \mathrm{~d}[6]$.

\section{1) Quasi linear inversion}

Find the disturbance quantity $m(x)$ to make the objective function reaches its minimum value,

$$
f(m)=\frac{1}{2}\|A(m)-d\|^{2} .
$$

the A denotes the nonlinear forward modeling operator, the $d$ represents the input data, the iterative equations are given by (15):

$$
m^{(k+1)}=m^{(k)}-\alpha L^{T}\left[A\left(m^{(k)}\right)-d\right],
$$

By equation (15) we can see that the linear inversion technology is similar to full waveform inversion. The differences in background model is precise enough premise, the migration operator in equation (15) depends only on the background model, and do not updating and changing in the iterative process [8].

2) Llinear inversion

Linear inversion compared with the similar linear inversion, we get the simulated data by linear operators L and $\mathrm{M}$ model and match the input data $\mathrm{d}$. The solution of linear equations:

$$
d=L m,
$$

The iterative solution:

$$
\begin{aligned}
& g^{(k)}=L^{T}\left[L\left(m^{(k)}\right)-d\right] \\
& \alpha=\frac{\left(g^{(k)}\right)^{T} g^{(k)}}{L\left(g^{(k)}\right)^{T} L g^{(k)}} \\
& m^{(k+1)}=m^{(k)}-\alpha g^{(k)}
\end{aligned}
$$

The $\alpha$ is analysis step, the $L^{T}$ is migration operator. The calculation of the above step mainly forward modeling operator and migration operator is the adjoint matrix based on the strict hypothesis. In actual processing,the two operators are difficult to achieve accurate, the the step size is usually not accurate [9-12].

\section{B. Numerical Simulation}

Marmousi model (Fig.3): mesh point is $151 * 521$, interval is $15 \mathrm{~m}$, offset is $20 \mathrm{~m}$, sampling rate is $6 \mathrm{~ms}$, record time is $3 \mathrm{~s}$; select the frequency range is $10 \sim 25 \mathrm{~Hz}$. 


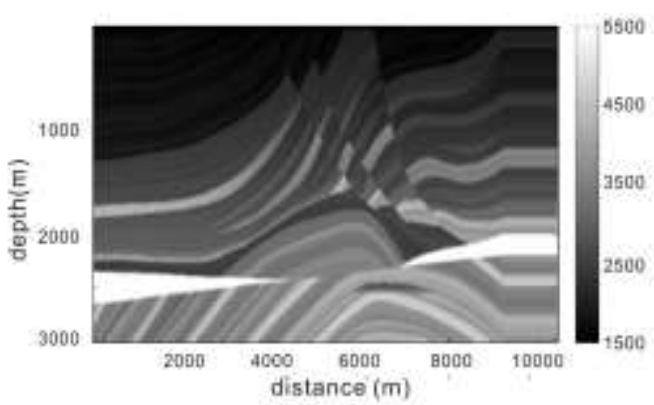

Figure 3. Marmousi model $(151 * 521)$

Using the six frequency, frequency domain migration of 120 guns, as shown in Fig .4. Because the selected band is less, so the migration imaging results in general.

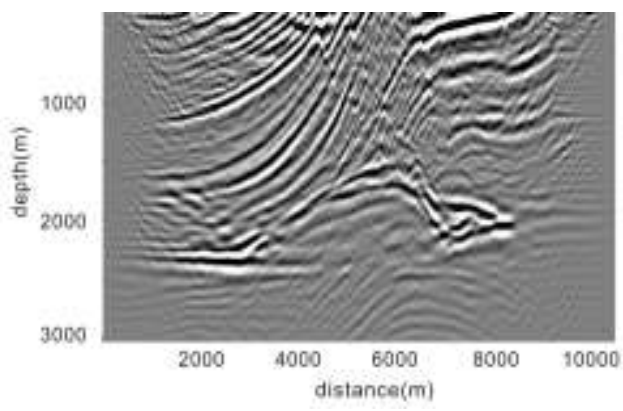

Figure 4. reverse time migration in frequency domain

Using the six frequency, frequency domain least squares inverse time migration of 120 guns, 10 iterations, as shown in Fig .5. From the figure we can get the imaging effect is obvious, the low-frequency interference opportunity not shallow.

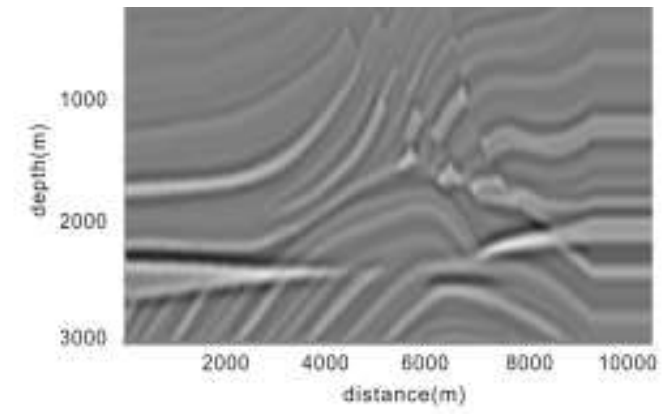

Figure 5. least squares reverse time migration in frequency domain ( 10 iterations )

Using six frequency, frequency domain least squares reverse time migration of 120 guns, after 20 iterations ,the results as shown in Fig .6. From the figure we can obtain the imaging effect more obvious, by low frequency interference shallow, and can be migration out of oil and gas under clearly.

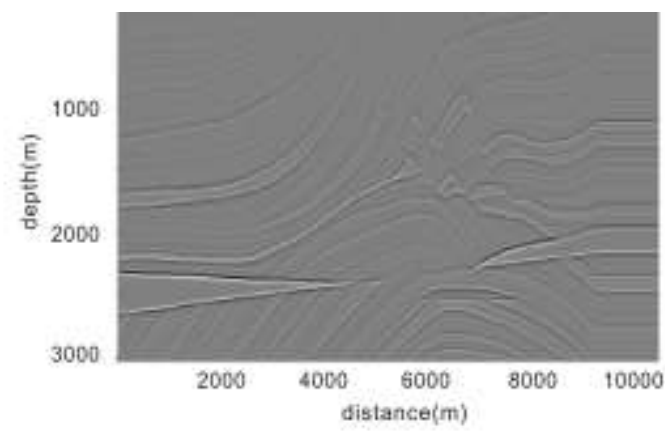

Figure 6. least squares reverse time migration in frequency domain (20 iterations )

Table 1 compares and analyzes the advantages and disadvantages of the frequency domain reverse time migration and the least squares reverse time migration.

TABLE 1

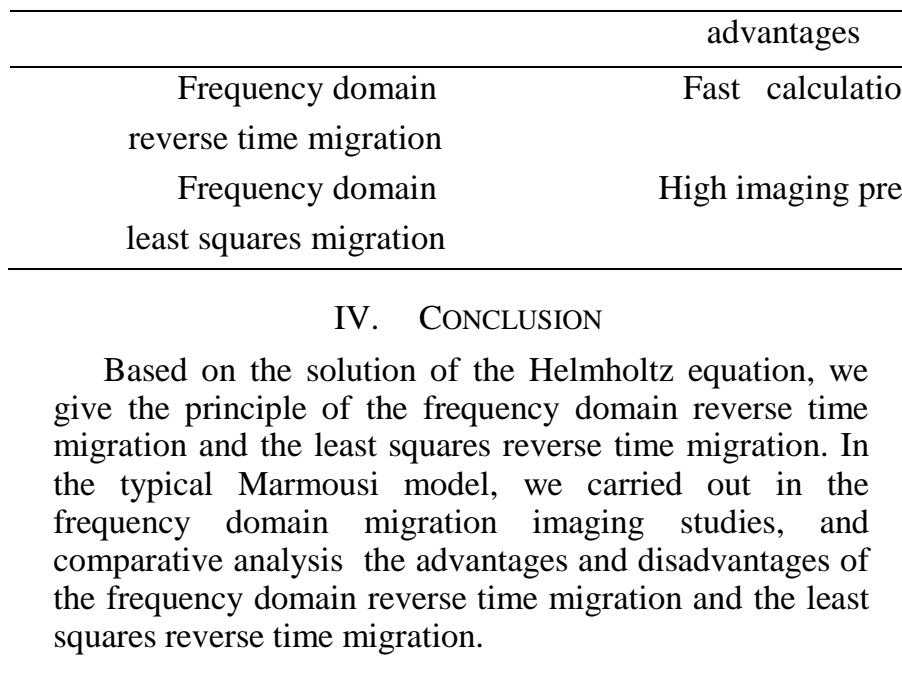

\section{REFERENCES}

[1] Gutton A, Kaelin B, Biondi B. Least square attenuation of reverse time migration artifacts[C]. 76th Ann. Internat. Mtg., Soc, Expl, Geophys., Expanded Abstracts, 2006(25): 2348-2352.

[2] Naoshi A, Schuster T G. Fast least-squares migration with deblurring filter [J]. Geophysics. 2009, 74(6): WCA83-WCA93

[3] Beasley C J, Chambers R E, Jiang Z. A new look at simultaneous sources[C]. 68th Annual International Meeting, SEG, Expanded Abstracts, 1998:133-135.

[4] Romero L A, Ghiglia D C, Ober C C, et al. Phase encoding of shot records in prestack migration[J]. Geophysics, 2000, 65(2):426-436.

[5] Ikelle L. Coding and decoding: Seismic data modeling, acquisition and processing[C]. 77th Annual International Meeting, SEG, Expanded Abstracts, 2007:51-55.

[6] Kwangjin Y, Kurt J. Challenges In Reverse-time Migration[C]. 74th Ann. Internat. Mtg., Soc, Expl, Geophys., Expanded Abstracts, 2004:1454-1458

[7] Liu Y K, Chang X, Jin D, et al,Reverse time migration of multiples for subsalt imaging[J]. Geophysics, 2011, 76( 5):WB209-WB216,

[8] Doulgeris P, Mahdad A, Blacquie're G. Iterative separation of blended marine data: discussion on the coherence-pass filter[C]. 
81th Annual International Meeting, SEG, Expanded Abstracts, 2011:26-31.

[9] Tan C, Han L, Zhang Y, Deng W. Separation of blended data by iterative denoising[C]. 74th Annual International Meeting, EAGE, Expanded Abstracts, 2012: A045.

[10] Wapenaar K,van der Neu J, Thorbecke J. Deblending by direct inversion[J]. Geophysics, 2012,77(3): A9-A12.
[11] Lynn W, Doyle M, Larner K, Marschall R. Experimental investigation of interference from other seismic crews[J]. Geophysics, 1987,52(2):1501-1524.

[12] Hampson G, Stefani J, Herkenhoff F. Acquisition using simultaneous sources[J]. The Leading Edge, 2008,27(7): 918-923. 